\title{
Solution to phenylbutazone purity challenge
}

\author{
Garnet McRae $^{1}$ - Donald M. Leek ${ }^{1} \cdot$ Enea Pagliano $^{1}$
}

(C) Her Majesty the Queen in Right of Canada as represented by: NRC Canada 2016

The joint winners of the phenylbutazone purity challenge (published in volume 408 issue 12) are: Lorenzo Biancalana and Beatrice Campanella, University of Pisa, Italy.

The award entitles the winning group to select a Springer book of their choice up to a value of $€ 100,-$.

Our Congratulations!

\section{Solution}

In the last 15 years NMR has become a reference technique for the determination of purity for organic standards containing NMR active nuclei [1, 2]. The puzzle proposed for this Analytical Challenge was focused on the use of ${ }^{1} \mathrm{H}-\mathrm{NMR}$ for the characterization of a phenylbutazone standard. Such material was purchased from Sigma-Aldrich and analyzed in parallel by HPLC-UV and ${ }^{1} \mathrm{H}-\mathrm{NMR}$. Initially, application of the two methodologies gave results which were not in agreement [3]. Whereas the HPLC-UV method found only two minor impurities that did not exceed the $1 \%$ level, the NMR assay was less generous: integration of the proton signal at position 5 (Fig. 1), provided a phenylbutazone purity estimate of only $0.8119 \pm 0.0014_{k=1} \mathrm{~g} / \mathrm{g}$ [3]. Such evidence could alert the analyst that an impurity, structurally related to phenylbutazone, is present. This

This article is the solution to the Analytical Challenge to be found at http://dx.doi.org/10.1007/s00216-016-9412-4

Enea Pagliano

enea.pagliano@nrc-cnrc.gc.ca;enea.pagliano@outlook.com

1 National Research Council of Canada, 1200 Montreal Road, K1A0R6 Ottawa, Canada conclusion would also be supported by the presence of an unusual signal at $2.2 \mathrm{ppm}$ in the ${ }^{1} \mathrm{H}$-NMR spectrum of phenylbutazone (Fig. 2) that should not be there. Such explanation suggests that something went wrong during the synthesis of the standard.

In this case, however, the guilty party is not the organic chemist, but the chemical character of phenylbutazone. Figure 1 summarizes the problem and solution for our case. Phenylbutazone undergoes keto-enol tautomeric equilibrium that makes the interpretation of the ${ }^{1} \mathrm{H}-\mathrm{NMR}$ data not very intuitive. The existence of this effect for phenylbutazone is reported in the literature [4] and is confirmed by the NOESY spectrum of phenylbutazone [3].

Since the keto form of phenylbutazone is in equilibrium with the enol, the proton in position 5 of the keto (chemical shift 3.9 ppm, Fig. 2) is shifted to the hydroxy group of the enol. This chemical effect makes the use of this proton unsuitable for quantitation purposes. Furthermore, the hydrogens in position 4 show a different chemical shift when the molecule is in a keto $(1.9 \mathrm{ppm})$ or enol form (2.2 ppm).

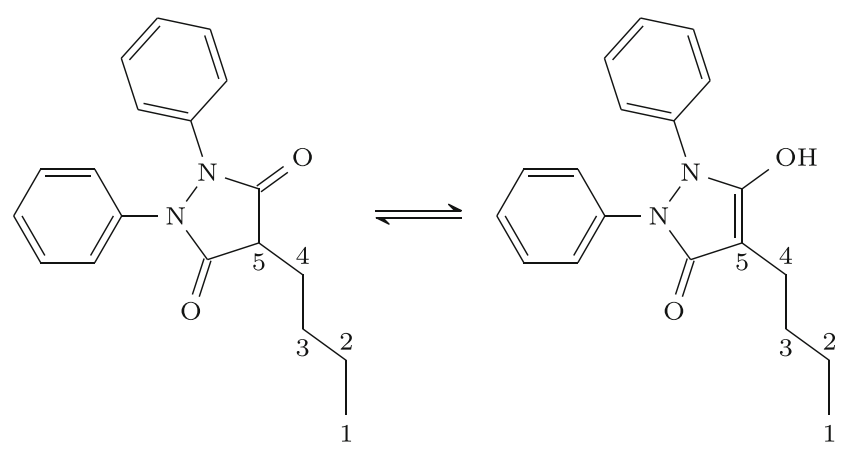

Fig. 1 Phenylbutazone keto-enol tautomerism 
Fig. $2{ }^{1} \mathrm{H}-\mathrm{NMR}$ spectrum of the phenylbutazone maleic acid mixture in DMSO- $\mathrm{d}_{6}$. When the phenylbutazone is in its keto form the hydrogens in position 4 can be seen at $1.9 \mathrm{ppm}$, whereas, when the molecule is in the enol, such hydrogens are detected as a triplet at $2.2 \mathrm{ppm}$

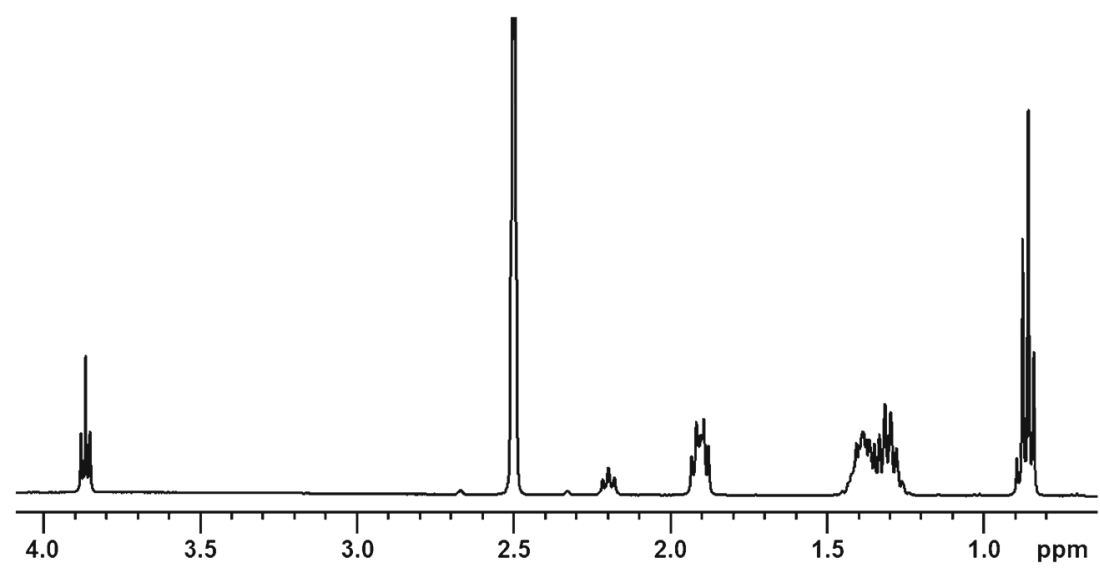

For the calculation of the purity, the analyst should opt for protons that are not affected by the tautomerism, such as those at $0.9 \mathrm{ppm}$ (belonging to the methyl group in position 1 ):

$$
\begin{aligned}
w_{\mathrm{A}} & =\frac{I_{\mathrm{A}}}{I_{\mathrm{B}}} \cdot \frac{N_{\mathrm{B}}}{N_{\mathrm{A}}} \cdot \frac{M_{\mathrm{A}}}{M_{\mathrm{B}}} \cdot \frac{m_{\mathrm{B}}}{m_{\mathrm{A}}} \cdot w_{\mathrm{B}}= \\
& =\frac{9.537}{10.000} \cdot \frac{2}{3} \cdot \frac{308.373}{116.0719} \cdot \frac{13.802}{23.332} \cdot 0.9995= \\
& =0.9987 \pm 0.0018 \mathrm{~g} / \mathrm{g}
\end{aligned}
$$

where $W_{i}, I_{i}, N_{i}, M_{i}$, and $m_{i}$ are respectively the purity (as mass fraction), NMR signal intensity, number of resonant nuclei, molar mass, and mass of the material $i$ (the phenylbutazone material is indicated with letter A, whereas the maleic acid internal standard with B). As an alternative to this calculation scheme, the analyst can utilize the signal at $1.3 \mathrm{ppm}$ which represents the four protons in positions 2 and 3:

$$
\begin{aligned}
w_{\mathrm{A}} & =\frac{12.705}{10.000} \cdot \frac{2}{4} \cdot \frac{308.373}{116.0719} \cdot \frac{13.802}{23.332} \cdot 0.9995= \\
& =0.9979 \pm 0.0018 \mathrm{~g} / \mathrm{g}
\end{aligned}
$$

In order to estimate measurement uncertainty, the JCGM guideline was applied [5]. NMR results are reported in

Table 1 Purity of phenylbutazone by ${ }^{1} \mathrm{H}-\mathrm{NMR}$

\begin{tabular}{llll}
\hline Signal ppm & $I_{\mathrm{A}}$ & $N_{\mathrm{A}}$ & $w_{\mathrm{A}} \mathrm{g} / \mathrm{g}$ \\
\hline 0.9 & $9.537 \pm 0.011$ & 3 & $0.9987 \pm 0.018$ \\
1.3 & $12.705 \pm 0.013$ & 4 & $0.9979 \pm 0.018$ \\
\hline
\end{tabular}

Experimental data: $I_{\mathrm{B}}=10.000 \pm 0.010_{k=1}, N_{\mathrm{B}}=2, w_{\mathrm{B}}=0.9995 \pm$ $0.0010_{k=1} \mathrm{~g} / \mathrm{g}, m_{\mathrm{A}}=23.332 \pm 0.003_{k=1} \mathrm{mg}, m_{\mathrm{B}}=13.802 \pm 0.003_{k=1}$ $\mathrm{mg}, M_{\mathrm{A}}=308.373 \pm 0.003_{k=1} \mathrm{~g} / \mathrm{mol}, M_{\mathrm{B}}=116.0719 \pm 0.0012_{k=1}$ $\mathrm{g} / \mathrm{mol}$.
Table 1 and they are in agreement with the purity obtained by HPLC-UV.

The apparent inconsistency between the two methodologies was simply due to the fast keto $\rightleftharpoons$ enol equilibrium (Fig. 1). Due to kinetics, the two tautomers could not be distinguished by conventional HPLC [6] and, therefore, only one major peak was detected. Despite this interesting chemical effect, the application of ${ }^{1} \mathrm{H}-\mathrm{NMR}$ - after $a$ proper interpretation of the chemistry - still produced a metrologically-sound purity estimation for phenylbutazone. The results presented in this Analytical Challenge further confirm that NMR is a powerful tool for purity assessment of organic compounds and a valuable asset for the production of Certified Reference Materials.

\section{References}

1. Pauli GF, Chen S-N, Simmler C, Lankin DC, Gödecke T, Jaki BU, et al. Importance of purity evaluation and the potential of quantitative ${ }^{1} \mathrm{H}$ NMR as a purity assay. J Med Chem. 2014;57:9220-31.

2. Malz F, Jancke H. Validation of quantitative NMR. J Pharmaceut Biomed Anal. 2005;38:813-23.

3. McRae $\mathrm{G}$ et al. The phenylbutazone purity challenge. Anal Bioanal Chem. 2016;408:3051-3.

4. Màczka P, Komsta Ł, Skibiński R, Gumieniczek A. Theoretical studies on keto-enol tautomerism, gas phase acidity and spectral properties of phenylbutazone. Ann Univ Mariae Curie-Skłodowska. 2010; 23:29-41.

5. Evaluation of measurement data - guide to the expression of uncertainty in measurement. JCGM 100, 2008.

6. Moriyasu M, Kato A, Hashimoto Y. Kinetic studies of fast equilibrium by means of high-performance liquid chromatography. part 11. keto-enol tautomerism of some $\beta$-dicarbonyl compounds. J Chem Soc Perkin Trans. 1986;2(4):515-20. 